\title{
Sloppiness in Spontaneously Active Neuronal Networks
}

\author{
Dagmara Panas, ${ }^{1 \star}$ Hayder Amin, ${ }^{2 *}$ Alessandro Maccione, ${ }^{2}$ Oliver Muthmann, ${ }^{1,3,4} @$ Mark van Rossum, ${ }^{1}$ \\ - Luca Berdondini, ${ }^{2} \uparrow$ and ${ }^{-}$Matthias H. Hennig ${ }^{\dagger} \dagger$ \\ ${ }^{1}$ Institute for Adaptive and Neural Computation, School of Informatics, The University of Edinburgh, Edinburgh EH8 9AB, United Kingdom, ${ }^{2}$ Istituto \\ Italiano di Tecnologia, Department of Neuroscience and Brain Technologies, 16163 Genoa, Italy, ${ }^{3}$ National Centre for Biological Sciences, Tata Institute of \\ Fundamental Research, Bangalore, Karnataka 560065, India, and ${ }^{4}$ Manipal University, Manipal 576104, India
}

\begin{abstract}
Various plasticity mechanisms, including experience-dependent, spontaneous, as well as homeostatic ones, continuously remodel neural circuits. Yet, despite fluctuations in the properties of single neurons and synapses, the behavior and function of neuronal assemblies are generally found to be very stable over time. This raises the important question of how plasticity is coordinated across the network. To address this, we investigated the stability of network activity in cultured rat hippocampal neurons recorded with high-density multielectrode arrays over several days. We used parametric models to characterize multineuron activity patterns and analyzed their sensitivity to changes. We found that the models exhibited sloppiness, a property where the model behavior is insensitive to changes in many parameter combinations, but very sensitive to a few. The activity of neurons with sloppy parameters showed faster and larger fluctuations than the activity of a small subset of neurons associated with sensitive parameters. Furthermore, parameter sensitivity was highly correlated with firing rates. Finally, we tested our observations from cell cultures on an in vivo recording from monkey visual cortex and we confirm that spontaneous cortical activity also shows hallmarks of sloppy behavior and firing rate dependence. Our findings suggest that a small subnetwork of highly active and stable neurons supports group stability, and that this endows neuronal networks with the flexibility to continuously remodel without compromising stability and function.
\end{abstract}

Key words: multielectrode array; network remodeling; neural culture; spike train analysis; statistical models

\section{Introduction}

Neuronal networks continuously remodel through changes in synaptic strength and number (Okabe et al., 1999; Holtmaat et al., 2005; Minerbi et al., 2009) and changes in neural excitability (Desai et al., 1999; Daoudal and Debanne, 2003). These changes are driven both by spontaneous activity (Maletic-Savatic et al., 1999; Okabe et al., 1999; Minerbi et al., 2009) and by experiencedependent plasticity during development and in the adult brain (Trachtenberg et al., 2002; Holtmaat et al., 2005). The presence of such changes is generally expected to destabilize stored information over time (Fusi and Abbott, 2007). However, functional representations in neural populations are consistently found to be very stable, despite continuous changes of the behavior of single neurons (Carmena et al., 2005; Chestek et al., 2007; Huber et al.,

\footnotetext{
Received Oct. 24, 2014; revised April 16, 2015; accepted April 22, 2015.

Author contributions: M.v.R., L.B., and M.H.H. designed research; H.A. and A.M. performed research; 0.M. contributed unpublished reagents/analytic tools; D.P. and M.H.H. analyzed data; D.P. and M.H.H. wrote the paper

This work was supported in part by grants EP/F500385/1 and BB/F529254/1 to the University of Edinburgh Doctoral Training Centre in Neuroinformatics and Computational Neuroscience from the UK EPSRC, BBSRC, and MRC research councils (D.P.), by the European Commission for Research within the Seventh Framework Programme for the NAMASEN (FP7-264872) Marie-Curie Initial Training Network (H.A.), the Istituto Italiano di Tecnologia (A.M., L.B.), the EurosSPIN Erasmus Mundus programme (0.M.), and the UK Medical Research Council Fellowship MRC G0900425 (M.H.H.). We thank Peter Latham for comments on a previous version of the paper.

${ }^{*}$ D.P. and H.A. contributed equally to this work.

TL.B. and M.H.H. contributed equally to this work as c0-senior authors.

The authors declare no competing financial interests.

Correspondence should be addressed to Dr Matthias H. Hennig, University of Edinburgh, 10 Crichton Street, Edinburgh EH89AB, UK. E-mail: m.hennig@ed.ac.uk.

DOI:10.1523/JNEUROSCI.4421-14.2015

Copyright $\odot 2015$ the authors $\quad 0270-6474 / 15 / 358480-13 \$ 15.00 / 0$
}

2012; Margolis et al., 2012; Ziv et al., 2013). These observations suggest that networks have considerable flexibility to reorganize structure without affecting previously established function. However, the relationship between plasticity of individual neurons and collective network stability is currently not well understood (Turrigiano, 2011).

Similar issues in systems biology relating to the interplay of robustness and evolvability were recently addressed by the theory of sloppiness in multiparameter models (Daniels et al., 2008). Within this framework, robustness of a model system stems from a highly anisotropic parameter space, where large regions of parameter values map onto very similar system behavior (sloppy parameters), and only few parameters or parameter combinations exert significant influence over collective activity (stiff parameters). Thus, local changes need not result in global changes, and global stability can be achieved through homeostatic regulation of only a few crucial parameters. A range of systems biology models have been found to indeed exhibit such properties (Brown and Sethna, 2003; Machta et al., 2013). Here we examine whether this concept can explain collective stability in networks of neurons.

We investigated this hypothesis using spontaneously active dissociated neural preparations, cultured on high-density multielectrode arrays. Such in vitro cultures are globally stable (Slomowitz et al., 2015) and at the same time undergo significant restructuring of synaptic connections (Maletic-Savatic et al., 1999; Okabe et al., 1999; Minerbi et al., 2009; Slomowitz et al., 2015), accompanied by changes in the activity of single neurons (Slomowitz et al., 2015). Importantly, these preparations allow 
for reliable recordings of hundreds to thousands of individual neurons over several days (Berdondini et al., 2009). We recorded from three hippocampal cultures and, similar to in vivo reports (Mizuseki and Buzsáki, 2013), we found highly skewed distributions of firing rates that were stable between consecutive recordings (Slomowitz et al., 2015). At the same time, the activity of single neurons and individual correlations changed substantially, indicating the presence of local fluctuations of neuronal properties.

To parametrically describe obtained multiunit spike trains, we used pairwise maximum entropy models (Schneidman et al., 2006; Shlens et al., 2006; Tang et al., 2008). This parametric form enabled us to use a recently introduced method to quantify the influence of parameter fluctuations on the collective network behavior (Machta et al., 2013). Interestingly, the model parameter sensitivity was highly uneven, implying sloppiness. This is, to our knowledge, the first demonstration of sloppiness in models directly fit to neuronal data. Our analysis further revealed that the most rapid and significant changes in neural activity occurred along the insensitive, sloppy parameter dimensions. In contrast, a small number of neurons, which had highly sensitive stiff parameters, exhibited slower and smaller fluctuations.

We further confirmed that also in vivo data and simulations of cortical activity in the asynchronous regime both exhibit sloppiness. Together, these results are consistent with the hypothesis that a small but stable subnetwork of neurons is responsible for global stability, allowing considerable plasticity to take place in the remaining majority of cells.

\section{Materials and Methods}

Dissociated cultures preparation. All procedures involving experimental animals were approved by the institutional IIT Ethic Committee and by the Italian Ministry of Health and Animal Care (Authorization ID 227, Prot. 4127 March 25, 2008). Neural cultures were obtained by dissociating hippocampal neurons from brain tissue of embryonic rats (both sexes) at day E18 and plating the cells (35,000-40,000 cells) on microelectrode arrays pretreated with adhesion factors (PEI/poly-L-lysine). Subsequently, cultures were incubated in Neurobasal supplement with $1 \%$ glutamax 2\% B-27 Neurobasal medium, in a humidified atmosphere $5 \% \mathrm{CO}_{2}$ at $37^{\circ} \mathrm{C}$. Fifty percent of the medium was changed every $3-4 \mathrm{~d}$. Three sets of recordings were obtained from three cultures from $24 \mathrm{~d}$ in vitro (DIV) onward over the course of 3-5 $\mathrm{d}$. As the recordings took place outside of the incubator, the dishes were closed with sterilized custom designed polydimethylsiloxane (PDMS) caps that prevent media evaporation and osmolarity changes while permitting gas exchange. After each recording, the chips were returned to the incubator after removal of the PDMS cap under a sterilized hood.

High-resolution multielectrode array recordings. High-resolution extracellular recordings were performed on the BioCam4096 platform with active pixel sensor multielectrode array (APS MEA) with chips type BioChip 4096S (3Brain), providing 4096 square microelectrodes of $21 \times$ $21 \mu \mathrm{m}$ in size on an active area of $2.67 \times 2.67 \mathrm{~mm}$ and with interelectrode separation of $21 \mu \mathrm{m}$. The platform records at a sampling rate of 7.022 $\mathrm{kHz}$ and 12 bits resolution per channel when measuring from the full $64 \times 64$ channel array. Raw data were visualized and recorded with the BrainWave software application provided with the BioCam4096 platform. Activity was recorded at 12 bits resolution per channel, low-pass filtered at $5 \mathrm{kHz}$ with the on-chip filter and high-pass filtered by a digital high-pass filter at $0.1 \mathrm{~Hz}$. Recordings, $15 \mathrm{~min}$ each, were performed at 0 , 2, 20, 50, 68, and $92 \mathrm{~h}$ in Culture 1 and at $0,2,20$, and $44 \mathrm{~h}$ in cultures 2 and 3 .

Spike detection. Spikes were detected with a custom algorithm (O. Muthmann, H. Amin, E. Sernagor, A. Maccione, D. Panas, L. Berdondini, and U. S. Bhalla, M. H. Hennig, unpublished data). In short, putative spikes were identified as negative voltage deflections crossing a specified threshold and satisfying simple signal shape constraints (mini- mum peak width of 2 frames, no other minimum in the following $1 \mathrm{~ms}$, partial repolarization). The threshold was defined in units of local noise estimate $v$ relative to baseline $b$ as follows: $b-5 v$. Baseline was defined as the local estimate of voltage tertile, incrementally updated in each time step. In a similar vein, the noise estimate was increased or decreased by a set value in each frame to reflect whether voltage was within $v$ of the baseline or not. Subsequently, a correlation-based analysis was performed to separate out the false-positives exploiting the random nature of random, uncorrelated electrode noise. To this end, the distribution of correlated channels for each putative spike was compared with a corresponding distribution of surrogate data of randomly generated spikes.

Because spike sorting on the full APS MEA system is not feasible due to the high neural density and limited acquisition rates, isolation of single units was not attempted. Given the small size of electrodes and limited signal spread between electrodes in in vitro cultures, each electrode was assumed to report the activity of a separate, single neuron, as was observed in previous work using cultured cortical neurons with comparable density (Berdondini et al., 2009). In rare cases of a spike appearing simultaneously ( \pm 1 frame) on neighboring electrodes, the lower amplitude event was ignored; however, such events were not common $(<3 \%$ in test recordings).

In vivo data. Single $15 \mathrm{~min}$ recording of in vivo activity of 140 neurons from primary visual cortex was obtained from the CRCNS.org on-line data repository, and was used here with permission from the authors. The dataset (Chu et al., 2014) and its description are available from the website. All experimental procedures are reported in detail in the original study (Chu et al., 2014). In short, a multishank multielectrode array (64 electrodes) was inserted normal to the cortical surface into the primary visual cortex (V1) of Macaca cyclopsis, and spontaneous activity was recorded under light anesthesia. Potential spikes were detected through filtering the recorded voltage $(400-5000 \mathrm{~Hz}, 28 \mathrm{Db} /$ octave $)$, digitalized and stored. Single units were isolated off-line using superparamagnetic clustering (Quiroga et al., 2004) with manual corrections.

Pairwise maximum entropy models. To avoid counting errors and extremely sparse pattern probability distributions, only channels $>0.1 \mathrm{~Hz}$ were considered. Channels active across all recordings from a single culture were identified and then 100 groups of 10 neurons each were randomly chosen for analysis. To this end, channel indices were permuted and first 10 neurons were chosen; channels that were selected more than four times were removed from further permutation runs. This procedure resulted in a homogeneous sampling over available population.

For each group, a pairwise maximum entropy model was fit to the data (Schneidman et al., 2006; Shlens et al., 2006; Tang et al., 2008). To this end, spike trains were binned, creating binary matrices $X=\left\{x_{j}^{t}\right\}$ with +1 representing a spike and -1 silence of unit $j$ in bin $t$. In each time bin, the state $X^{t}$ of the group could be 1 of $2^{10}$ possible spiking patterns $X_{s}$ (e.g.; $1,-1,1,1,-1,1,-1,1,1,1)$. The fraction of how often state $X_{s}$ occurred in the data yielded the probability distribution of spiking patterns in the group $P_{o b s}\left(X_{s}\right)$. This distribution will typically depend on the choice of a time bin, as larger time bins are more likely to include more simultaneous spikes and thus exhibit more synchrony, possibly including spurious higher-order correlations. On the other hand, too small time bins will ignore existing and pertinent synchrony. The particular choice we made in this work was dictated by consideration of the time scale of latency of a monosynaptic connection. As reported previously (Fitzsimonds et al., 1997; Erickson et al., 2008), the majority of latencies between a presynaptic and postsynaptic spike in a dissociated hippocampal culture fall within 1-5 ms. We therefore chose a time bin of $5 \mathrm{~ms}$.

The distribution obtained from the data can be approximated by a maximum entropy model under first and second order constraints, i.e., a model that reproduces the following marginals:

$$
\begin{gathered}
\left\langle x_{j}\right\rangle=\frac{1}{T} \sum_{t=1}^{T} x_{j}^{t}, \\
\left\langle x_{j} x_{k}\right\rangle=\frac{1}{T} \sum_{t=1}^{T} x_{j}^{t} x_{k}^{t},
\end{gathered}
$$


and is otherwise unconstrained, i.e., is maximized with respect to entropy. The model that satisfies those criteria takes the parametric form of the Boltzmann distribution:

$$
P\left(X_{s}\right)=\frac{\exp (-H)}{\sum_{\left\{X_{s}\right\}} \exp (-H)},
$$

where $H$ is a measure closely related to energy:

$$
H=\sum_{j} \lambda_{j} x_{j}+\frac{1}{2} \sum_{k \neq j} \lambda_{j k} x_{j} x_{k}
$$

with parameters, $\lambda_{j}$ and $\lambda_{j k}$, describing the functional excitability and the functional interactions of neurons. To obtain a model fit, the parameters were adjusted by an iterative scaling algorithm as follows:

$$
\begin{gathered}
\delta \lambda_{j}=\eta \times \operatorname{sign}\left(\left\langle x_{j}\right\rangle_{o b s}\right) \times \ln \left(\frac{\left\langle x_{j}\right\rangle_{o b s}}{\left\langle x_{j}\right\rangle_{o b s}}\right), \\
\delta \lambda_{j k}=\eta \times \operatorname{sign}\left(\left\langle x_{j} x_{k}\right\rangle_{o b s}\right) \times \ln \left(\frac{\left\langle x_{j} x_{k}\right\rangle_{o b s}}{\left\langle x_{j} x_{k}\right\rangle_{o b s}}\right) .
\end{gathered}
$$

A constant learning rate $\eta<1$ was used for each set of neurons. Adjustments continued until the relative difference between model and observed marginals was on the order of $10^{-5}$, which corresponds to reproducing spike and coincident spike numbers with the precision of $\sim 1$ spike. If a group did not converge within a prescribed number of iterations (typically 50,000), alternative value of $\eta$ was used, to obtain the desired convergence. In the rare cases of incomplete convergence, the precision dropped to $\sim 1.5-2.5$ spikes.

Comparison measures. To compare the pattern probability distributions between consecutive days and between the model and the data, the Jensen-Shannon divergence was used:

$$
\begin{aligned}
D_{J S}\left(P_{1} \| P_{2}\right)=\frac{1}{2} \sum_{\{X\}} P_{1}(X) \log _{2}( & \left.\frac{2 P_{1}(X)}{P_{1}(X)+P_{2}(X)}\right) \\
& +\frac{1}{2} \sum_{\{X\}} P_{2}(X) \log _{2}\left(\frac{2 P_{2}(X)}{P_{1}(X)+P_{2}(X)}\right) .
\end{aligned}
$$

To compare group parameters between time points while allowing for overall fluctuations in excitation or coupling, we used the coefficient of determination $r^{2}$. To this end, for two sets of parameter values of a group $i, \Lambda_{i, t_{1}}=\left\{\lambda_{j}^{i}, \lambda_{j k}^{i}\right\}_{t_{1}}$ at time $t_{1}$ and $\Lambda_{i, t_{2}}$ at time $t_{2}$, linear regression was performed, fitting the following:

$$
\Lambda_{i, t_{2}}=a \times \Lambda_{i, t_{2}}+b .
$$

The $r^{2}$ of this fit was used as a measure of similarity between parameter groups. The comparisons were calculated in reference to the baseline recording, unless otherwise noted.

Fisher information matrix. The Fisher information matrix (FIM) for a probabilistic model with observables $x$, and parameters $\Lambda=\left\{\lambda_{l}\right\}$ is defined as follows:

$$
f_{l m}(\Lambda)=-\sum_{x} p(x \mid \Lambda) \frac{\partial \ln p(x \mid \Lambda)}{\partial \lambda_{l}} \frac{\partial \ln p(x \mid \Lambda)}{\partial \lambda_{m}} .
$$

In the particular case of a pairwise maximum entropy model, $l, m \epsilon\{j, j k\}$, and so for 10 neurons the FIM has the following form:

$$
F=\left(\begin{array}{cccccc}
f_{1,1} & \ldots & f_{1,10} & f_{1,\{1,2\}} & \ldots & f_{1,\{9,10\}} \\
f_{2,1} & \ldots & f_{2,10} & f_{2,\{1,2\}} & \ldots & f_{2,(9,10)} \\
\vdots & \ddots & \vdots & \vdots & \ddots & \vdots \\
f_{\{9,10\}, 1} & \ldots & f_{\{9,10\}, 10} & f_{\{9,10\},\{1,2\}} & \ldots & f_{\{9,10\},\{9,10\}}
\end{array}\right) .
$$

The FIM provides a measure of the curvature of the log-likelihood landscape with respect to the model parameters, and as such it quantifies the sensitivity of the model to parameter changes. For the pairwise maximum entropy model the matrix entries can be directly computed from the model, taking on the form of the covariance matrix of the random variables associated with all parameters in turn:

$$
F_{l m}(\Lambda)=\left\langle X_{l} X_{m}\right\rangle_{\text {mod }}-\left\langle X_{l}\right\rangle_{\text {mod }}\left\langle X_{m}\right\rangle_{\text {mod }},
$$

where $X_{l}=x_{j}$ for the parameters $\lambda_{j}$ and $X_{\mathrm{m}}=x_{j} x_{k}$ for the couplings $\lambda_{j k}$. The matrix can also be computed from the data, by taking expectations with respect to the measured probability distribution. Direct comparison showed that these two versions of the FIM were almost identical. Moreover, for a statistical physics model such as Equation 3, the FIM can equivalently be expressed as the free entropy change of a system with respect to model parameters, which has the Riemannian property of a metric tensor (Crooks, 2007).

Gini coefficient analysis. The Gini coefficient is a commonly used and robust (Hurley and Rickard, 2009) measure of sparsity. For a distribution of $N$ values $f_{i}$, it is defined as follows:

$$
G=1-2 \sum_{i=1}^{N} \frac{f_{i}}{\sum f_{i}}\left(\frac{N-i+1 / 2}{N}\right) .
$$

As a control, we also computed Gini coefficients for two artificially generated maximum entropy models. The first was a maximum entropy model with will all interactions $\lambda_{j k}$ set to 0 , and the fields $\lambda_{j}$ set to a value that reproduces the firing rates of the data (Culture 1 , recording at $0 \mathrm{~h}$, and in vivo recording). This model provides an estimate of sparsity under the assumption of an independent model, where neurons do not interact, and the number of relevant parameters is reduced from 55 to 10 . The second model used was an entirely artificial 'toy' model, with fields and interactions set as follows

$$
\begin{aligned}
\lambda_{j}^{\text {homog }} & =0.14+r_{j} \frac{\operatorname{var}\left(\lambda_{j}\right)}{10}, \\
\lambda_{j k}^{\text {homog }} & =0.14+r_{k} \frac{\operatorname{var}\left(\lambda_{j k}\right)}{10},
\end{aligned}
$$

where $r_{j}$ and $r_{j k}$ were randomly drawn numbers from a uniform distribution, variance was set to one-tenth of that of the fitted models, and the mean was arbitrarily chosen. This model was aimed to provide an example of how nonsparse a maximum entropy model can be if the fields and interactions are homogeneous, and therefore there is little differentiation in their sensitivity.

Shuffling and resampling procedures. To verify that presented results are not due to overfitting data of limited sample size nor random effects, shuffling and resampling procedures were used to generate surrogate data for comparisons. In the shuffling procedures neurons or groups of neurons (see Figs. $2 E, F, 5 B$, respectively) had their indices permuted before computing comparison measures against original, unshuffled data. For group shuffling, this was repeated $N_{\text {shuf }}=50$ times. In the resampling procedure, for each group of neurons an artificial spike matrix $X=\left\{x_{j}^{t}\right\}$ was generated by randomly sampling from the model distribution $P_{\text {mod }}(X)$. Subsequently, a pairwise maximum entropy model was fit to the artificial data, exactly as for the observed spike trains. This was repeated $N_{\text {res }}=50$ times. Fisher Information Matrices were calculated, and eigendecompositions were conducted as for the data, and the resulting resampled FIMs, parameters, and eigenparameters could then be compared against the original true data in parallel to comparisons between recordings. The differences against original data were averaged across resamples in each group.

Network simulations. Four separate networks of 3200 excitatory and 800 inhibitory randomly connected leaky integrate and fire neurons were simulated for $900 \mathrm{~s}$. The resting potential was set to $-49 \mathrm{mV}$, the membrane time constant to $20 \mathrm{~ms}$, the spike threshold to $-50 \mathrm{mV}$ and the reset potential to $-60 \mathrm{mV}$. Current-based synapses were modeled as low-pass filters with time constants $5 \mathrm{~ms}$ for excitatory and $10 \mathrm{~ms}$ for inhibitory connections. The weight of inhibitory synapses was set such that it resulted in an IPSP of $-9 \mathrm{mV}$. The weight of the excitatory synapses was different for each of the four networks, resulting in EPSPs of 

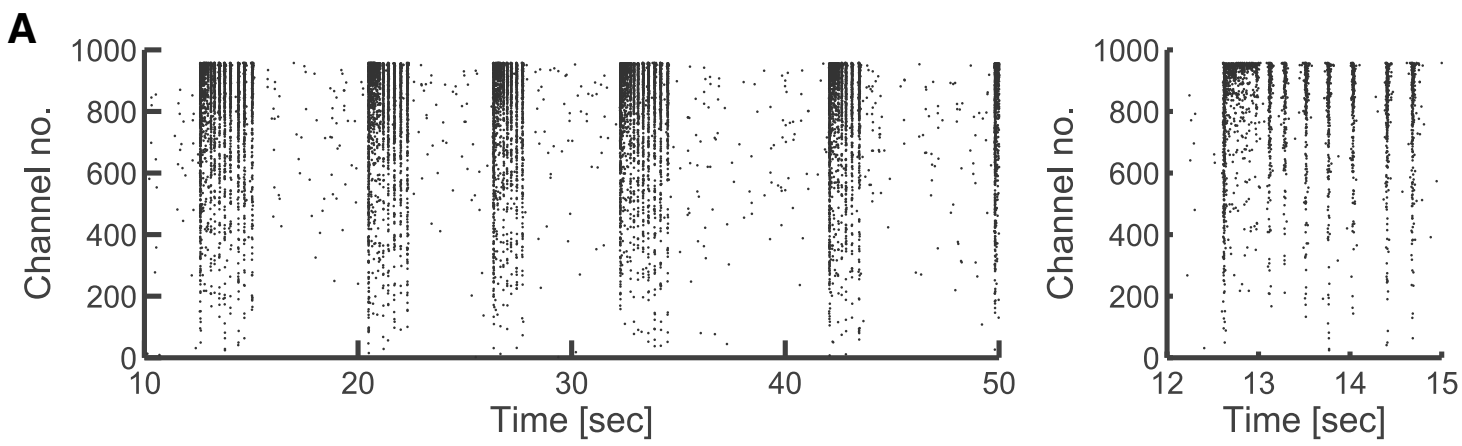

B

Oh

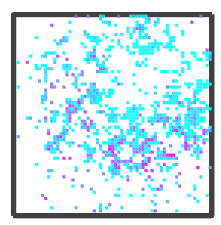

$2 \mathrm{~h}$

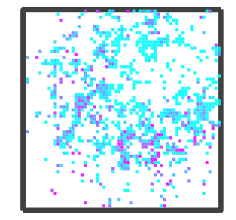

$20 \mathrm{~h}$

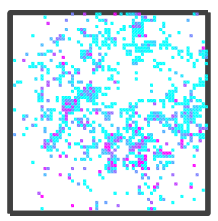

$50 \mathrm{~h}$

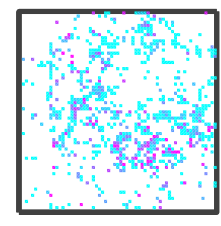

$68 \mathrm{~h}$

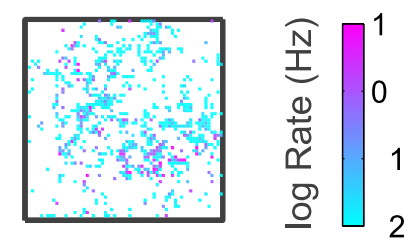

C

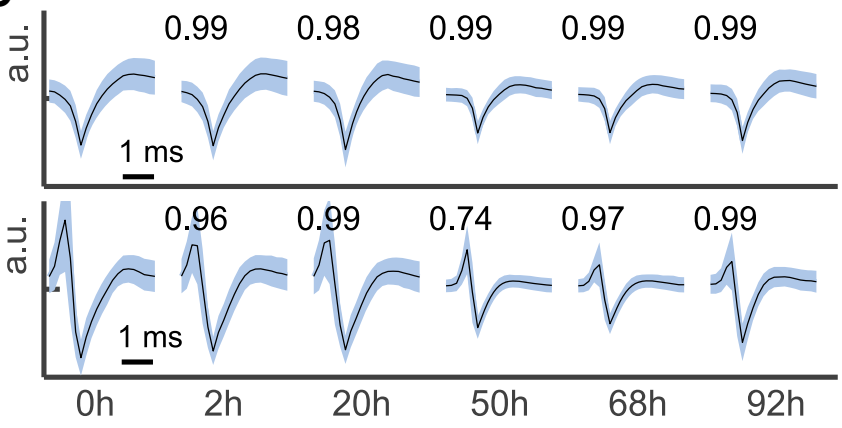

D

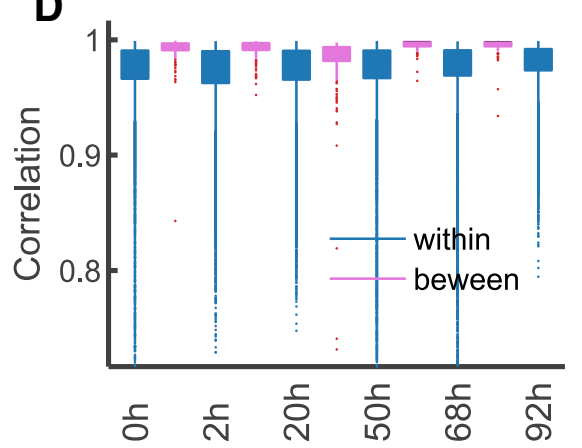

Figure 1. High-resolution recordings from cultured neurons and monitoring single units over time. $A$, Example raster plot of culture activity (left), and a close-up of a superburst (right); all channels $>0.01 \mathrm{~Hz}$ shown for illustration. $\boldsymbol{B}$, Distribution of firing rates on the array monitored over time (color-coded log firing rate of each channel arranged on a $64 \times 64$ lattice corresponding to the physical layout of the MEA); all channels $>0.01 \mathrm{~Hz}$ shown for illustration. C, Example spike shape voltage traces of two electrodes at different time points; mean (black) and SD (pale blue), averaged across detected spikes within a recording. Overlaid are values of the correlation coefficients between the presented mean spike shapes. $\boldsymbol{D}$, The distributions (median and interquartile ranges and outliers) of spike-shape correlation values between consecutive recordings in Culture 1 (pink); only channels $>0.1 \mathrm{~Hz}$ shown, as used in the main analysis. Shown for comparison (blue) are the distributions of correlations between average spike shapes of different units within a recording.

1.6, 2, 2.25, and $2.5 \mathrm{mV}$ for "lif 1"- "lif 4," respectively. The networks were randomly initialized with voltages from a uniform distribution ranging from -60 to $-50 \mathrm{mV}$. These simulations were run with the Brian Simulator v1.4.1 (Goodman and Brette, 2009). Randomly selected groups of neurons were analyzed as described above.

Code and data availability. The code for reproducing the results shown in the paper, as well as a sample dataset, are provided online as a GitHub repository at: https://github.com/dpanas/SloppyIsing.

\section{Results}

Neural activity from cultured dissociated hippocampal neurons was recorded with a high-density 4096 channel MEA (Fig. 1A,B) starting from the $24 \mathrm{DIV}$, where synaptogenesis and other developmental processes are complete (Lesuisse and Martin, 2002; Wagenaar et al., 2006). Fifteen-minute-long recordings were taken from three cultures at different time points over several days (Fig. 1B). Intervals between recordings ranged from 2 to $30 \mathrm{~h}$, a time scale on which spontaneous synaptic remodeling was reported in cultured neurons (Minerbi et al., 2009). Typically, 1500-3500 channels reported spiking activity, with most neurons spiking at rates $<1 \mathrm{~Hz}$. Because estimation of spike shapes, rates, and correlations is unreliable for low spike rates, we restricted our analysis to units with rates $>0.1 \mathrm{~Hz}$, leaving between 400 and 1600 viable channels.
As established in previous research (Berdondini et al., 2009), the APS MEA allows for stable recordings from single units, due to high density and small size of the electrodes, and the typical shunt resistance in high-density cultures. To further verify that the single-unit resolution was preserved across time, we compared average spike shapes between consecutive recordings. As depicted in Figure 1C, even the least correlated waveforms (bottom) appear to retain their characteristic features across recordings (despite the fact that a media change at $42 \mathrm{~h}$ affected all channels and reduced signal strength across the array). Furthermore, examining the distribution of the correlation coefficients between spike shapes shows that waveforms are highly correlated between recordings, significantly more so than waveforms from different electrodes (Fig. 1D).

The activity showed the typical spontaneous, nonrandom bursting patterns observed in mature cultures (Segev et al., 2004; van Pelt et al., 2005; Wagenaar et al., 2006), with 50-100 short and highly synchronized bursts per minute, and almost no spiking between bursts (Fig. $1 A$ ). Notably, although the appearance of highly synchronous bursts might suggest otherwise, pairwise correlations were generally weak (Fig. 2A). At a fine time resolution of $5 \mathrm{~ms}$, on which synaptic events take place (Fitzsimonds et 
al., 1997; Erickson et al., 2008), the median correlation coefficient was $\sim 0.05$ or less (Fig. 2B), and correlation matrices were dense and unstructured. The presence of these weak yet significant correlations motivated the use of maximum entropy models in this study (Schneidman et al., 2006; Shlens et al., 2006).

Dense culture recordings are stable despite significant individual fluctuations

First we examined the overall stability of the population activity over time. Firing rate distributions remained largely unchanged from day to day in all preparations (Fig. $2 A, B$ ), as indicated by a Kolmogorov-Smirnov test between consecutive pairs of recordings ( $p$ values, Culture 1: 0.298, 0.464, 0.851, 0.18, 0.609; Culture 2: 0.064, 0.027, 0.516; Culture 3: $0.028,0.004,0.075)$. Pairwise correlations were less stable, but always significantly rank-correlated from recording to recording (average Kendal tau correlation coefficient 0.51 , ranging from 0.37 to $0.69)$. In contrast to the overall population activity, the activity of single neurons showed clear and significant changes, as illustrated in Figure 2C. Between two recordings, individual firing rates could increase or decrease by $>100 \%$ (relative to the first), with most changes approximately $\leq 25 \%$ and one-quarter of the population changing by $>50 \%$. These firing-rate changes revealed a tendency of slowly firing neurons to increase their activity, and of neurons with high rates to decrease activity (Fig. 2D). This bias became more pronounced over time and was consistent over different preparations. Importantly, this could not simply be accounted for by random fluctuations, as the estimated variance of firing rates in single channels was significantly lower than the population variance estimated from shuffled data, particularly for neurons with higher firing rates (Fig. 2E,F). Pairwise correlations between neurons changed even more prominently (often one-half of the correlations changed by $>50 \%$ ), but less consistently than firing rates when compared over time and across preparations. Together, these results demonstrate the presence of significant fluctuations in the activity of single neurons, although overall the networks maintained stable firingrate distributions, in accordance with other reports (Slomowitz et al., 2015).

Changes in populations of neurons

To analyze the impact of single-neuron fluctuations on group behavior, we turned to a parametric model of multineuron activity. Pairwise maximum entropy models were fit to a 100 randomly selected groups of 10 neurons. Groups were selected from
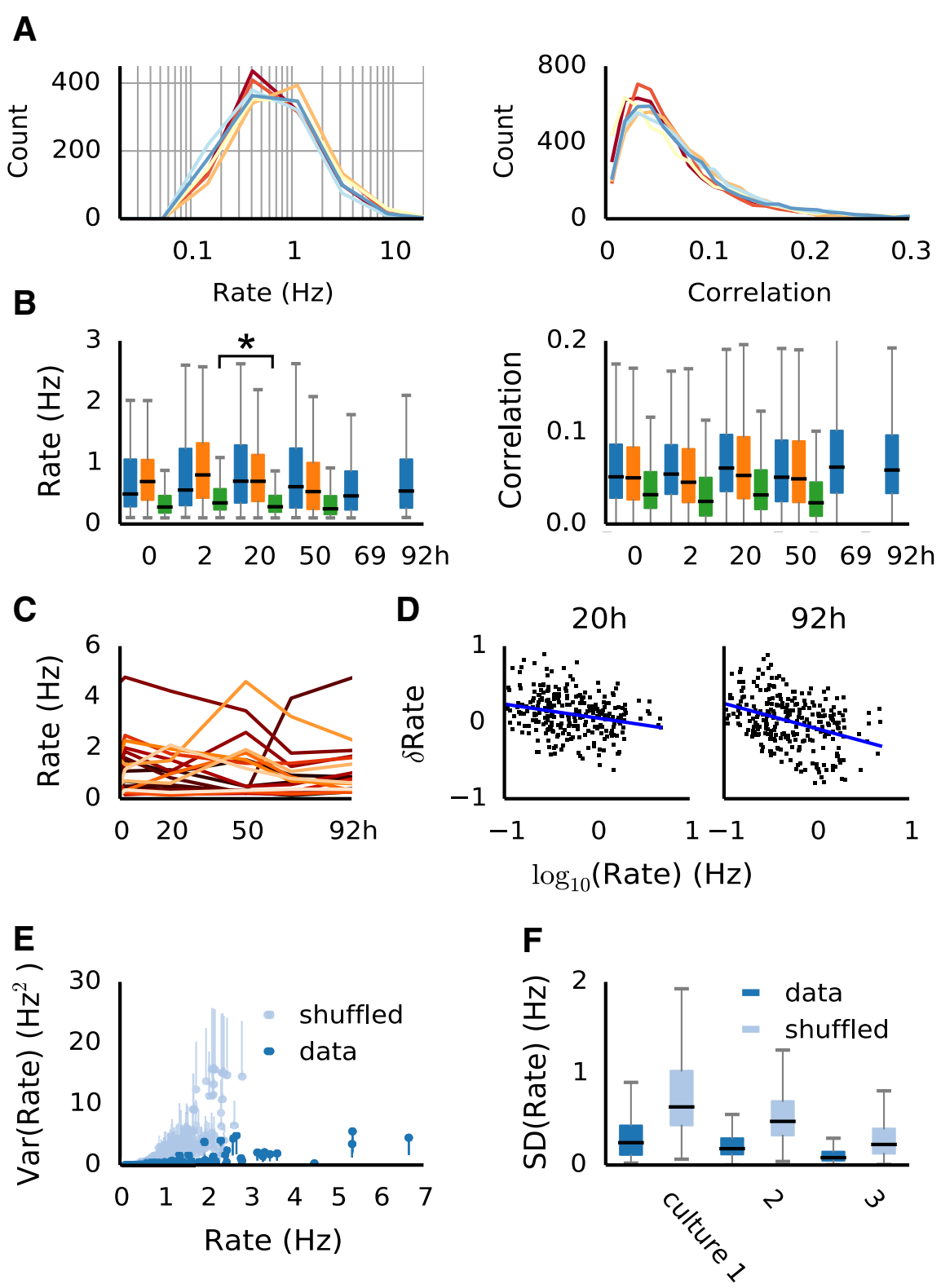

Figure 2. The distributions, but not individual firing rates are preserved over several days in neural cultures. $\boldsymbol{A}$, Stable distributions of firing rates (left; Kolmogorov-Smirnov test reported no significant differences at $\alpha=0.05$ ) and similar distributions of pairwise correlations (right; significantly correlated between recordings at $p<0.01$ ) in a single preparation over several days. $\boldsymbol{B}$, Distributions (median and interquartile ranges) of firing rates (left) and correlations (right) in all three preparations. Firing rate distributions remained stable (Kolmogorov-Smirnov test yielded $p>0.05$ in 8 , and $p>0.01$ in 10 of the 11 pairs; the outlier pair marked by an asterisk). C, Example of firing rates across recordings of 20 randomly selected individual neurons. D, Rate changes are not entirely random, but exhibit a bias of neurons with low firing rate to increase activity, and neurons with higher activity to decrease firing (blue lines). Graphs show the relative changes, $\delta$ Rate $=\left(R_{t_{2}}-R_{t_{1}}\right) /\left(R_{t_{1}}-R_{t_{2}}\right)$ between time points $t_{1}$ and $t_{2}$. $\boldsymbol{E}$, The relationship between the mean firing rate and the variance of firing rate in individual neurons across recordings (blue dots; error bars shown only on one side for clarity). For comparison, the same is plotted for shuffled data (gray; unit rates were randomly shuffled within recording), as an estimate of the behavior of population variance. $\boldsymbol{F}$, Distributions (median and interquartile ranges) of firing rate SD of individual neurons, compared with shuffled data, for all recordings.

channels with activity in all recordings, to allow for systematic comparison across an entire experiment. These models aim to reproduce the probabilities of each pattern of silence and spiking by fitting a Boltzmann distribution with matching first and second order marginals, where each group $i$ has a set of parameters $\Lambda_{i}$ : local biases $\lambda_{j}$, controlling independent spiking of each neuron $j$; and interactions $\lambda_{j k}$, representing functional couplings between neurons $j$ and $k$, required to model their joint firing. The models captured on average $70-90 \%$ of the multineuron corre- 

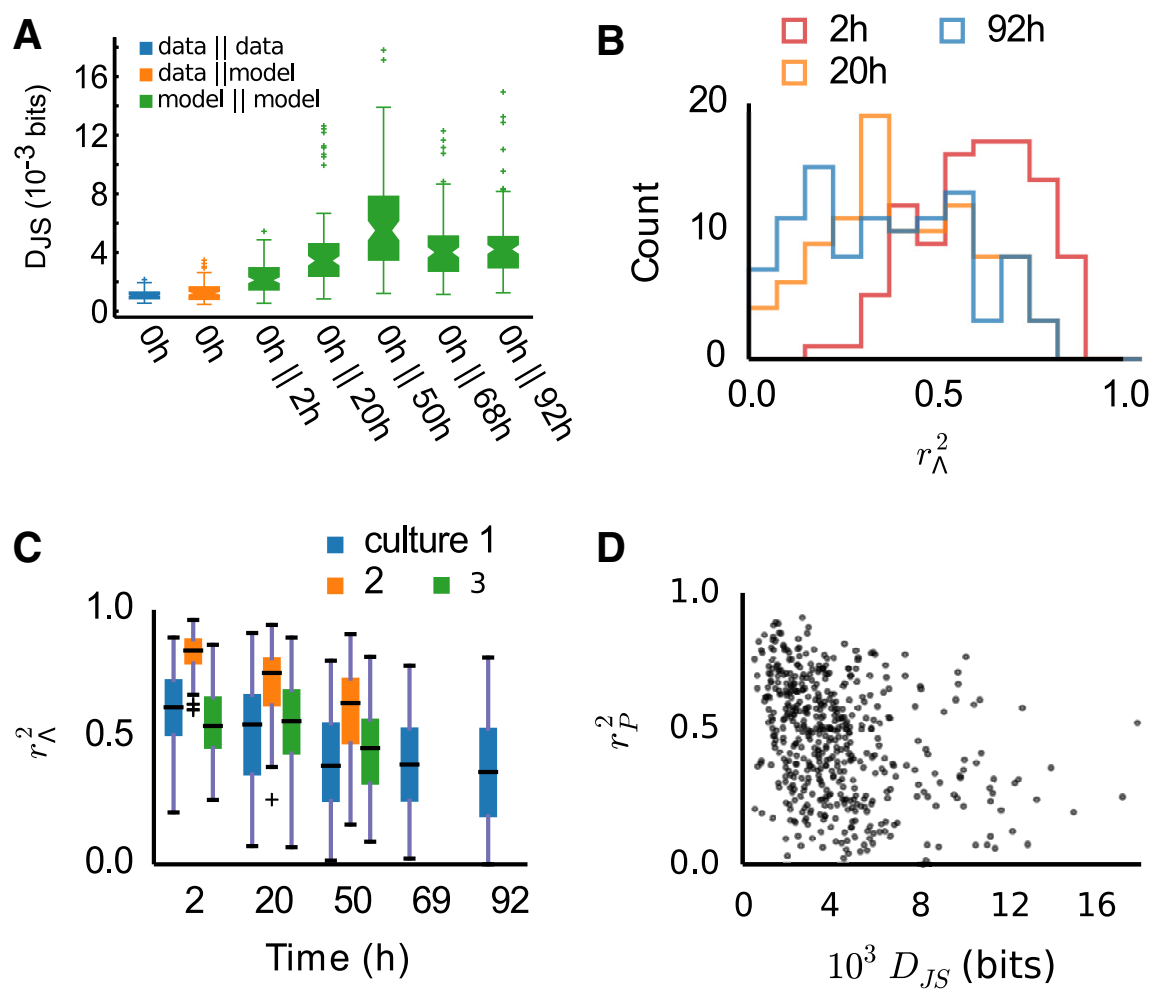

Figure 3. Pairwise maximum entropy models reflect changes in network activity over time. $A$, Jensen-Shannon divergence $D_{\mathrm{JS}}$ between models at different time points from the same neuron groups. The probability distributions predicted by the models became increasingly dissimilar over time. For reference and indication of model fit quality, also shown are representative $D_{\text {Is }}$ between probability distributions obtained from two halves of a single recording, and between model and data. $\boldsymbol{B}$, Distributions of model parameter similarity $r_{\Lambda}^{2}$ between recordings at different time points, in a single preparation. For longer intervals, the differences between the models become more pronounced. $\boldsymbol{C}$, Summary of the average parameter similarity at different time points, for all preparations. $\boldsymbol{D}$, Parameter similarity between recordings is not predictive of distribution similarity; a scatterplot of $r_{\Lambda}^{2}$ of each group against the $D_{\mathrm{Js}}$ of the same group.

lation structure, similar to reports from other studies (Schneidman et al., 2006; Tang et al., 2008; Ohiorhenuan et al., 2010). Furthermore, comparison of Jensen-Shannon divergences shows that the model predicts the data on a comparable level with prediction of one-half of the dataset from the other half (Fig. $3 A$ ).

We then proceeded to compare the models between each recording taken at different time intervals. Over time, activity distributions became increasingly dissimilar, as measured by the Jensen-Shannon divergence $D_{\mathrm{IS}, i}$ (Fig. $3 A$ ). To assess singleneuron fluctuations, we directly compared the model parameters of each group $i$ between time points, using the coefficient of determination $r^{2}$ of the linear regression between the parameter values of each group at two different time points (see Materials and Methods). In-line with the results shown so far, significant and increasing changes between recordings were apparent as a decreasing average and broadening distribution of $r_{\Lambda, i}^{2}$ values (Fig. $3 B, C$ ). Yet, there was only a weak relationship between parameter dissimilarity and $D_{\mathrm{IS}, i}\left(r^{2}<0.15, p<0.05\right.$; Fig. $\left.3 D\right)$. This result is due to the nonlinear relationship between singleneuron parameters and multineuron group behavior, and illustrates that the local changes do not predictably translate into global changes.

Sloppiness in populations of neurons and implications for stability To understand the effects of local changes and systematically examine their impact on model similarity, we used the FIM. The FIM measures the curvature of the model log-likelihood with respect to the parameters, thus characterizing the parameter sen- sitivity of the model. Additionally, it provides a true metric for the model class used here, and therefore enables a direct comparison of models between recordings (for details, see Materials and Methods).

In a first step, we obtained the FIM for each group of neurons (Fig. 4A, second panel), and following the approach of Machta et al. (2013), we performed eigenvector decomposition of the FIMs (Fig. $4 A$ ). Because the form of the FIM is such that each entry corresponds to a pair of parameters (see Materials and Methods), the matrix does not map in a straightforward manner onto parameters themselves. However, in analogy with principal component analysis of a covariance matrix, an eigenvector decomposition of FIM can be performed to obtain the dimensions in parameter space providing the dominant contribution to the matrix. For sloppy models, this eigendecomposition should result in rapidly decreasing eigenvalues, reflecting the anisotropy of the model space and revealing the sloppy (insensitive to changes) and stiff (sensitive) dimensions in the space of parameters. As in other sloppy systems (Daniels et al., 2008; Machta et al., 2013), we observed rapidly decreasing eigenvalues (Fig. $4 B$, representative example, $D$, average behavior). The principal eigenvector explained on average $\sim 80 \%$ of the variance (Fig. $4 E$ ), hence yielding the most relevant contribution to parameter sensitivity and defining the stiffest direction in parameter space. On the other hand, eigenvectors associated with low eigenvalues correspond to sloppy dimensions, along which parameters can change without significantly affecting the spike pattern distributions (Fig. $4 F$, illustrative example). Thus we obtained a remapping of parameter space that allowed us to project the parameter vectors of each model onto eigenvectors and examine whether the observed changes between recordings happen along sloppy or stiff dimensions (see next section; Fig. 5D).

To further examine the parameter space anisotropy, and in particular to assess how many parameters were relevant to the stiff dimensions in our models, we turned to sparsity analysis. This was motivated by the fact that a FIM that is both sloppy and sparse indicates that the model is sensitive to only a small number of model parameters and their combinations. In contrast, a FIM that exhibits sloppiness but not sparseness indicates that the stiff dimensions involve more complicated combinations of many parameters. Therefore, as a measure of sparseness, we computed Gini coefficients (Hurley and Rickard, 2009) for each FIM. To provide a frame of reference, we also calculated Gini coefficients for sets of FIMs obtained from models without interactions and models with more homogeneous activity than the data. The first set came from maximum entropy models with null interactions and fields set to reproduce the firing rates of the data. This created much sparser FIMs than the data (Fig. 4C, "indep"), and provided an estimate of the upper limit of sparsity given the observed firing rates. The second set was from an Ising model with param- 
A

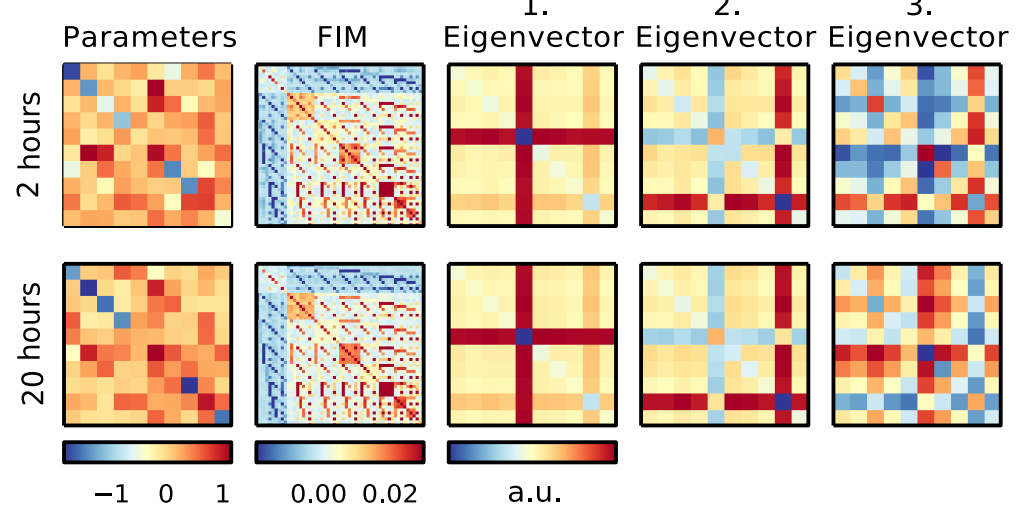

B

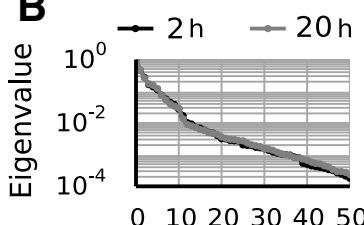

E

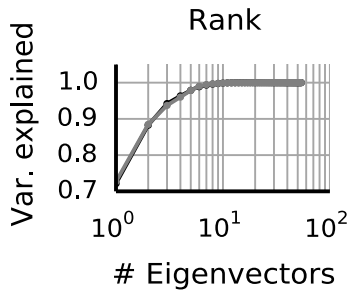

\# Eigenvectors

C
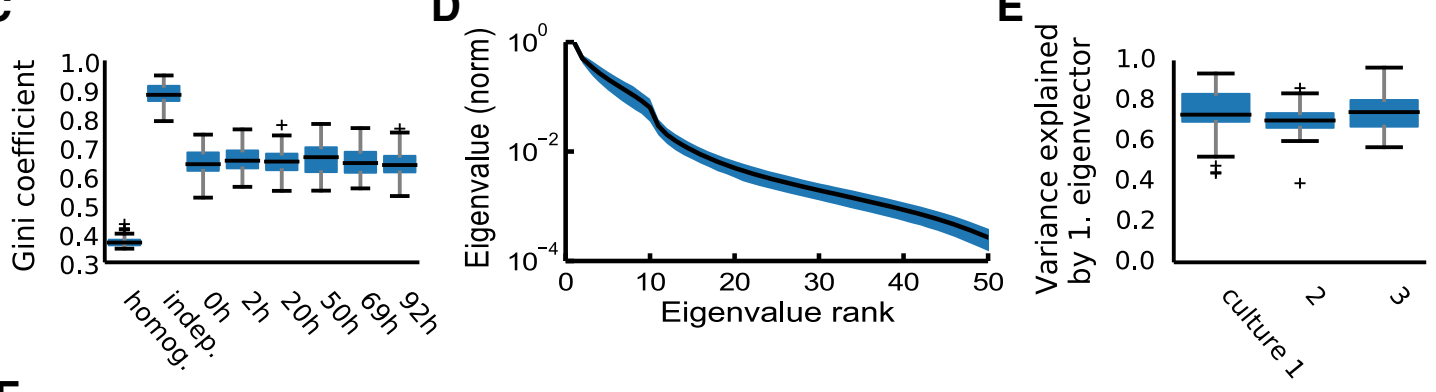

F
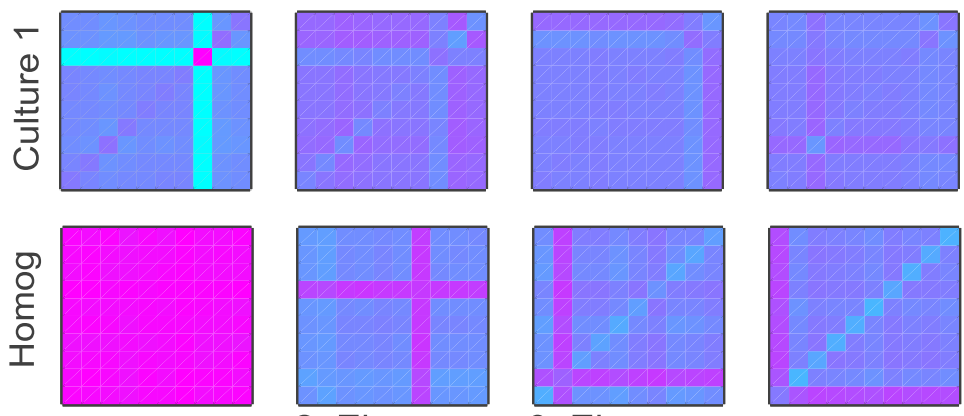

1. Eigenvec

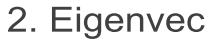

4.Eigenvec

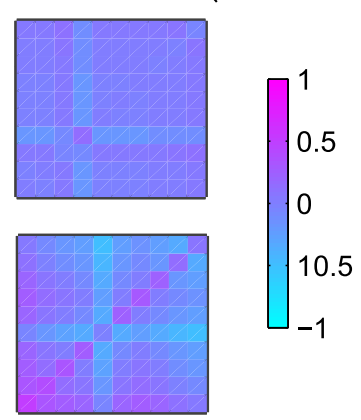

5.Eigenvec

Figure 4. The FIM of maximum entropy models reveals a sloppy parameter space for neural activity. $\boldsymbol{A}$, For each model (left; diagonal elements are local fields, upper triangle represents interactions, lower triangle filled to visualize that couplings between neurons are symmetric) the FIM is computed (second left). FIM is a $55 \times 55$ matrix, as there are 55 independent parameters in the model, 10 fields, and 45 symmetrical interactions. Next, the FIMs eigenvalues and eigenvectors are determined (middle to right; arrangement corresponding to the arrangement of parameters). This example shows the same group of neurons recorded $18 \mathrm{~h}$ apart (top vs bottom row). Although the parameters differ, the two FIMs and their first two eigenvectors are almost indistinguishable. For these two models, $D_{\mathrm{IS}}\left(M_{1} \mid M_{2}\right) \approx 0.007, r_{E}^{2}=0.99$ for the principal eigenvector and $r_{\Lambda}^{2}=0.61$ for the parameters. $\boldsymbol{B}$, Eigenvalues of the example two models (top) and fraction of FIM variance explained by adding consecutive eigenvectors (bottom). $\boldsymbol{C}$, Gini coefficients for the FIM computed for nonsparse Ising models (homogeneous; local fields and interactions both drawn from normal distributions with mean 0.14 , and variance $1 / 10$ of fitted fields and interactions), independent models (matching the firing rates of the data), and from six datasets recorded at different time points (same preparation as $\boldsymbol{A}$ ). $\boldsymbol{D}$, The shape of average eigenvalue distribution across groups in the first recording of Culture 1 , with SD in blue. Note, log scale used for the eigenvalues; the disparity between eigenvalues and fast saturation of explained variance both suggest that the models are sloppy. $\boldsymbol{E}$, FIM variance explained by the principal eigenvector across all groups in each of the cultures (first recording only, as it is representative). $\boldsymbol{F}$, The first five eigenvectors (scaled by eigenvalues relative to the first) of a random group of neurons from Culture 1 (top row), against first five eigenvectors of a random group of neurons from the toy homogeneous model (bottom row).

eters more homogeneous than the fitted models (see Materials and Methods), to illustrate that sparsity is not an intrinsic feature of this model class. This resulted in very uniform FIMs (Fig. $4 C$, "homog"), reflecting a hypothetical scenario where all neurons' fields and interactions are very similar, and therefore little differentiation of sensitivity is expected. For our data-fitted models, the FIMs of all groups across all time points were always significantly sparser than those of the homogeneous models (Fig. 4C). This indicates that many parameters in our models do not contribute to sensitivity and therefore do not contribute to the stiff dimensions. This is illustrated in Figure $4 F$, where only a subset of parameters defines the principal eigenvector, in contrast to what occurs in a homogeneous model. On the other hand, a significantly lower FIM Gini coefficient compared with independent indicates that the sparsity is not simply due to a smaller number of parameters where the interactions are irrelevant.

\section{Networks change faster along insensitive dimensions}

Having established that our models of neural activity are sloppy, we then proceeded to examine whether sloppiness contributes to stability. First, a comparison of FIMs between recordings indicated that, despite substantial differences in model parameters and resulting observables, the sensitivities of the models remained relatively stable, with median coefficient of determination remaining $>0.7$ even for an interval of $92 \mathrm{~h}$ (Fig. $5 A, B$, compare with Fig. $3 B, C$; a case where the FIM is identical over an interval of $18 \mathrm{~h}$ is illustrated in Fig. 4A). At the same time, the activity of groups of neurons slowly changed over the recordings, an effect that clearly exceeded noise 
A

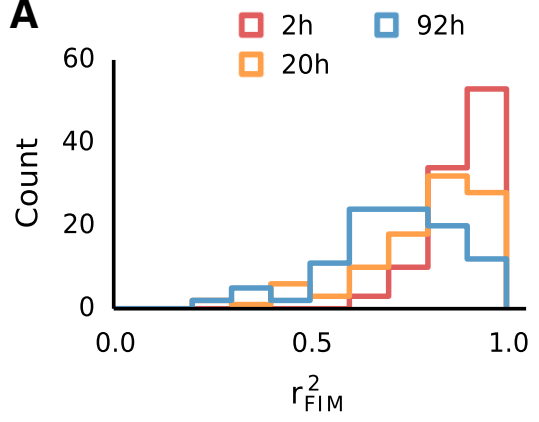

B

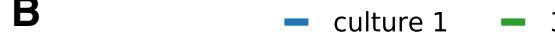

C

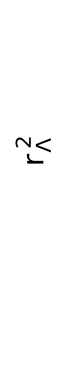

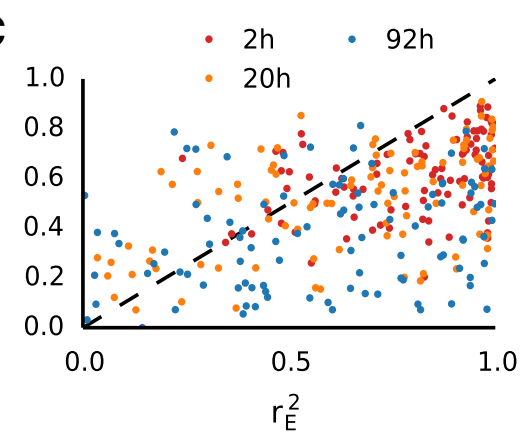

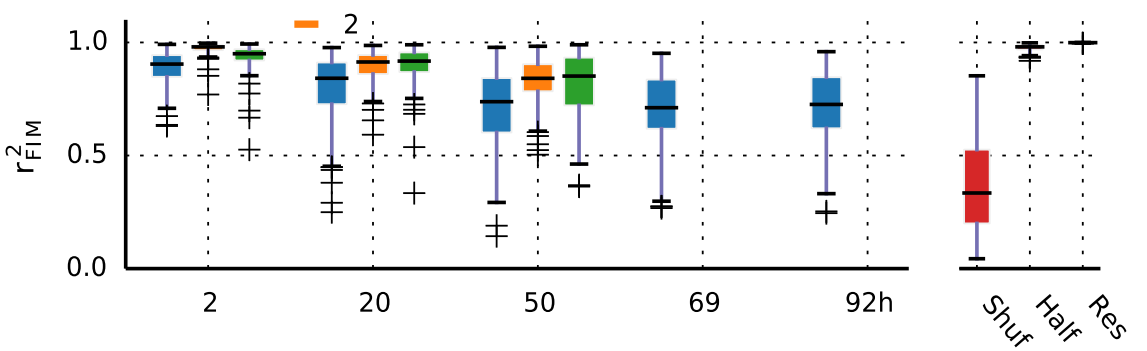

D
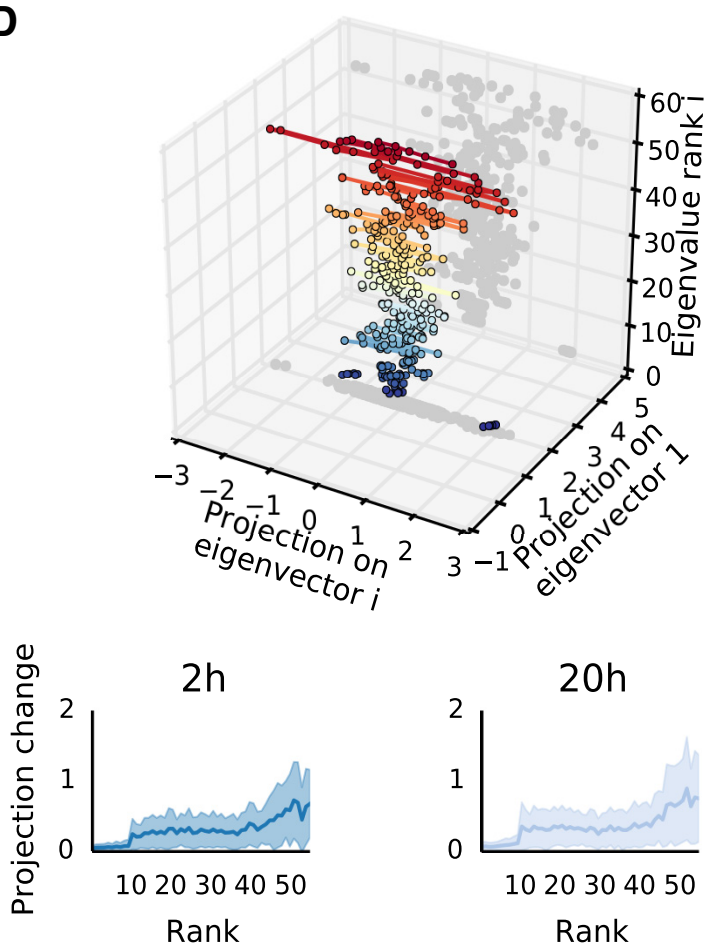

Figure 5. Fluctuations in network parameters predominantly occur in sloppy dimensions with weak influence on collective dynamics. $\boldsymbol{A}$, Distributions of FIM similarity $r_{F I M}^{2}$ for different intervals (Culture 1). Note much higher similarity than for model parameters. B, FIM similarities $r_{F I M}^{2}$ for all experiments at different intervals. For comparison, $r_{F I M}^{2}$ is also shown for shuffled groups (Shuf), for models fit independently to two halves of the same recording (Half) and to models fit to data resampled from previously fit models (Res). C, Comparison of parameter similarity in each group, versus eigenparameter similarity, for three pairs of recordings in Culture 1.D, Eigenvector decomposition allows for subspace projections for each group's parameters. Here a single group is imaged across six recordings (same group as $A, B$ ). Projections onto theprincipal andi't theigenvector arein thex-yplane, and thez-axis denotestherankioftheeigenvector (also indicated bydifferent colors). Dots represent projections of one parameter setat different time points. These become progressively wider with increasing eigenvector rank, indicating stronger changes along sloppy dimensions. Right, Scatter-plots of projections between two time points, pooled across all groups, in the same color code as the 3D figure. Bottom, The average magnitude of change in parameter projections as a function of eigenvector rank.

levels estimated by cross-validation or resampling from models (Figs. 3A, 5B), evident both in pattern distributions and FIMs. However, examining the projections of parameters onto the eigenvectors (Fig. 5D) revealed that the majority of changes between recording
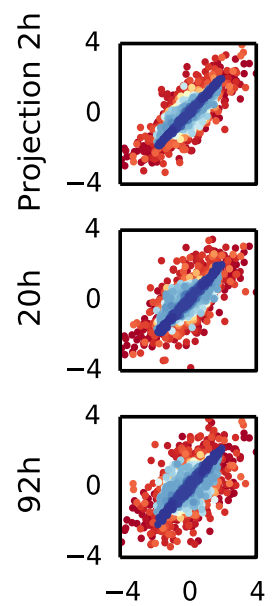

Projection $\mathrm{Oh}$

$92 \mathrm{~h}$

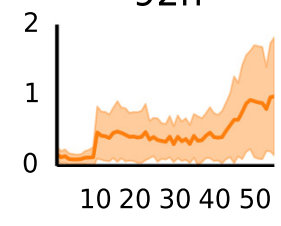

Rank sessions occurred along the sloppy dimensions in parameter space (Fig. 5D). Scatterplots of parameter projection values from a given time point against the baseline (Fig. $5 D$, right) indicate that the projections onto stiff dimensions (blue) remain nearly unchanged, whereas the most pronounced changes are confined to projections onto the sloppiest dimensions. In line with this, the average (across groups) of the absolute projection changes clearly shows an increase with eigenvector rank (Fig. 5D, bottom).

To provide a complimentary view on the relationship between changes and sloppiness, and to be able to link parameter sensitivity to direct neural measures, we conducted further analysis, focusing on the stiffest dimension. Because the dominant eigenvector informs us of the relative contribution of individual parameters to the stiff dimension, and those contributions are sufficiently differentiated, we adopted the principal eigenvector entries (referred to as eigenparameters, $v_{i j}$ ) as sensitivity measures of corresponding parameters. We then analyzed the relationship between parameter sensitivity and changes in the activity, parameters, and eigenparameters of corresponding neurons. As shown in Figure $6 A, B$ (top), the largest fluctuations consistently occurred along the least sensitive dimensions in model parameter space. These parameter changes were accompanied by corresponding changes in spike counts, which were stronger for insensitive associated parameters, at least for short intervals (Fig. 6A, $B$, middle). However, this relationship became less prominent after $2 \mathrm{~d}$, suggesting that slow network remodeling took place. This is in line with decreasing similarity of model distributions (Fig. 3A) and FIMs (Fig. $5 A, B$ ), as these results indicate that on longer time scales increased remodeling takes place. To further understand this phenomenon, we also examined the behavior of eigenparameters over time. As for the parameters, here initially the changes occurred mostly in insensitive regions, but after the second day concerted and significant changes became apparent in sensitive regions as well (Fig. $6 A, B$, bottom). A comparison with shuffled data demonstrates that the trend we observe could not be explained by random effects (Fig. $6 B$, red lines), and modeling resampled data indicates no influence of uncertainties in parameter estimation from finite amounts of data (Fig. 6B, light green lines).

Altogether, these results indicate that stability between recordings is associated with stability in sensitive neurons. Furthermore, we also found a tight positive relationship between parameter sensitivity and firing rates of the corresponding neu- 


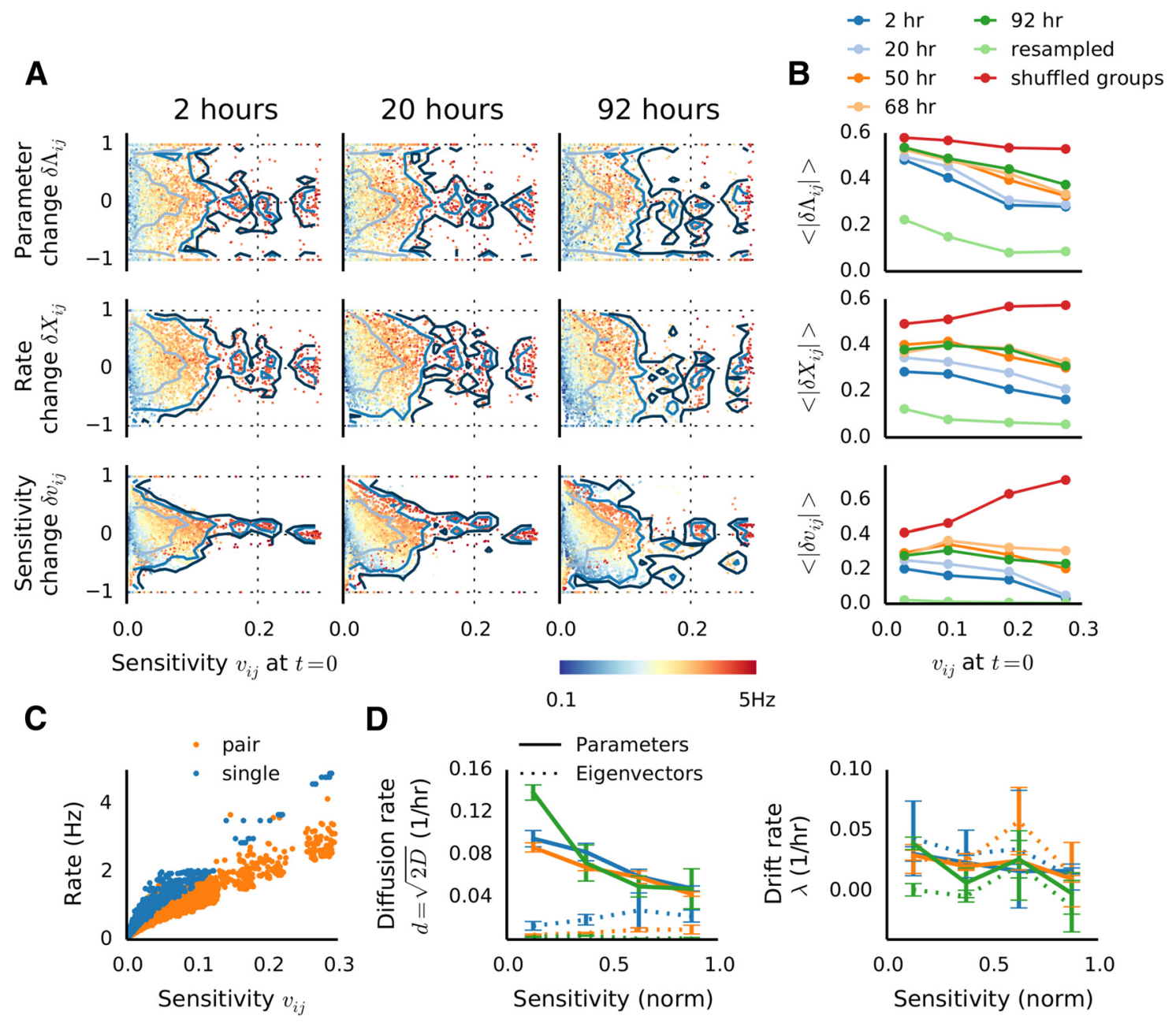

Figure 6. Sensitivity to changes, high-firing neurons, drift and diffusion. $A$, Relative changes of model parameters (top, $\delta \Lambda_{i j}$ ), data marginals (middle, $\delta \mathrm{X}_{i j}$ ) and eigenparameters (bottom, $\delta v_{i j}$ ) at different time points in one experiment, plotted as a function of the eigenparameters (which are used here as a measure of parameter sensitivity). Marginals were estimated as the spike counts and counts of coincident spikes. Relative changes were calculated as $\delta=\left(A_{2}-A_{1}\right) /\left(\left|A_{1}\right|+\left|A_{2}\right|\right)$. Colors indicate the average firing rate of each neuron pair at the corresponding time points. Contour lines indicate 1,2, and 3SD. B, Degree of relative change of parameters (top), marginals (middle), and eigenparameters (bottom), expressed as the absolute mean of the distributions in $\boldsymbol{C}$, as binned into quarters according to the sensitivity. For comparison, resampled data (light green) and shuffled data (dark red). $\boldsymbol{C}$, The relationship between firing rate and sensitivity shows a clear dependence of sensitivity on the firing rate of corresponding neuron(s). D, Ornstein-Uhlenbeck model parameters estimated for the parameters (straight lines) and eigenparameters (dashed lines). Diffusion coefficients $d$ (left) and drift constants $\lambda$ (right) were estimated by fitting $\operatorname{Var}\left(\Delta J_{i j}\right)_{\Delta t}=\frac{D}{\lambda}\left(1-e^{-2 \lambda \Delta t}\right)$, where $D$ is the diffusion constant and $\lambda$ the drift rate. The curves were generated by dividing the sensitivity into four bins, and normalizing the range to enable comparison across recordings. Error bars indicate SEM of the least-squares fit.

ron pairs (Fig. 6A, colors, $C$ ); in all preparations, the correlation between those measures was $>85 \%$, and in most $>91 \%$. This implies that highly active neurons generally have a disproportionally strong influence on the collective network activity, much more so than functionally strongly coupled neurons, as there was no significant relationship between parameter sensitivity and correlations. We further explored the notion of high-firing units influence by conducting additional sparsity analysis. To this end, we again randomly sampled groups of neurons, this time however restricting the choice of units to those with firing rates $>1$ Hz. As shown in Figure 7E, FIMs of high-firing groups were significantly less sparse than those of random groups. This indicates that sensitivity is more uniform for groups comprised solely of highly active neurons.

Finally, to obtain a quantitative estimate of the rates of the parameter and eigenparameter fluctuations, we turned to their description by a mean-reverting stochastic process. Because their distributions were stable over time and the variance of their fluc- tuations closely approximated $\operatorname{Var}\left(\frac{x_{t_{2}}-x_{t_{1}}}{\Delta t}\right) \propto(\Delta t)^{-2}$ (not illustrated), an Ornstein-Uhlenbeck, which combines diffusion with a mean-reverting drift, is an appropriate model. This model has been used before to describe synaptic weight dynamics (van Rossum et al., 2000; Minerbi et al., 2009; Loewenstein et al., 2011), and enabled us to estimate quantities characterizing the fluctuations, and their dependency on parameter sensitivity.

We estimated the diffusion rate $\delta=\sqrt{2 D}$, where $D$ is the diffusion constant, and the drift rate $\lambda$ separately for the coupling parameters, and corresponding eigenparameters for four ranges of sensitivities. Consistent with the results shown so far, diffusion was fastest for insensitive and slowest for the most sensitive parameters (Fig. $6 D$, left, straight lines). In contrast, and consistent with the small effect of parameter fluctuations along sloppy dimensions, the diffusion rate for eigenparameters was significantly smaller (Fig. $6 D$, left, dashed lines). The drift rates, on the 
A
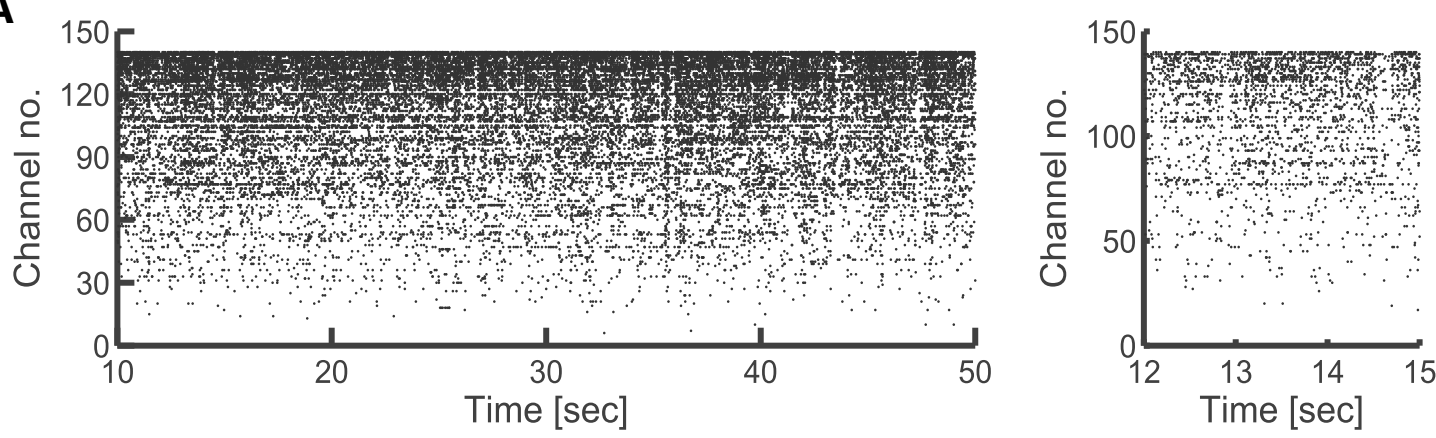

B
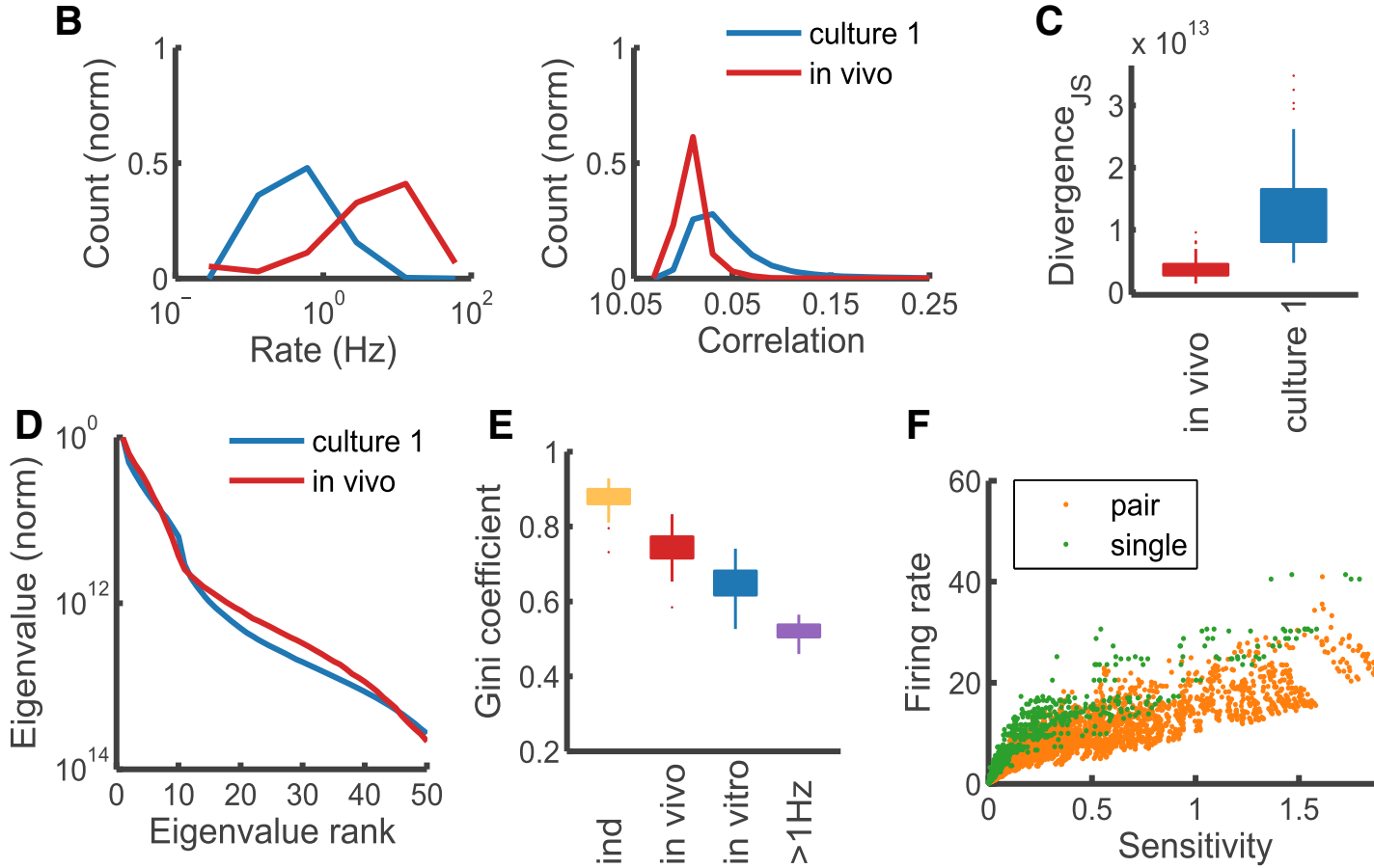

$\mathbf{F}$

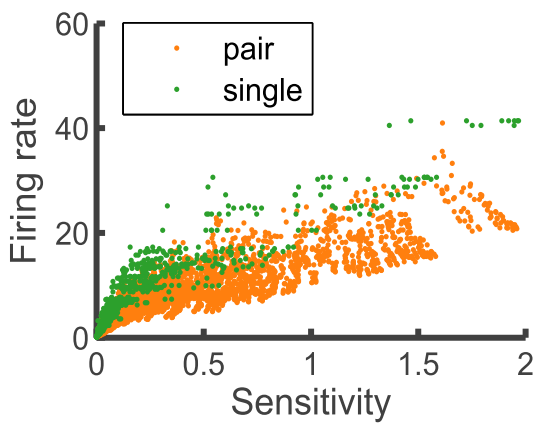

Figure 7. Spontaneous activity of in vivo neurons is sloppy and sparse. $\boldsymbol{A}$, Example raster plot of spontaneous in vivo activity recorded from primary visual cortex of macaque monkey. $\boldsymbol{B}$, Distributions of firing rates and correlations in the spontaneous cortical activity, compared with those of in vitro data (first recording, Culture 1). C, Jensen-Shannon divergence $D_{J 5}$ between model and the data, compared with same from in vitro data (first recording, Culture 1). D, Comparison of the average eigenvalue distributions (scaled to the first eigenvalue, averaged across all groups) between in vivo and in vitro models. E, Comparison of the distributions of model sparsity between different models. "Ind," Independent model reproducing the firing behavior of in vivo data; "in vivo," pairwise model fit to in vivo data; "in vitro," example of pairwise model fit to in vitro data (first recording, Culture 1); ">1 Hz," pairwise model fit to in vitro data filtered by firing to include only channels $>1 \mathrm{~Hz}$. F, Relationship between parameter sensitivity and the firing rate ("single" denotes the "field" parameter, "double" refers to the "interaction" parameter between two neurons; for a pair of neurons the firing rate is the average of the two).

other hand, showed a weak dependency on sensitivity, were similar for parameters and eigenparameters, and consistently smaller than the diffusion rates, and are therefore likely associated with actual network remodeling (Fig. 6D, right).

\section{Sloppiness and sparsity in vivo}

The above analysis of in vitro recordings suggests sloppiness as a candidate mechanism contributing to stability in networks of neurons, organizing neuronal activity such as to not rely uniformly on all network parameters. However, although cultured neurons might reveal innate tendencies of self-organizing neural circuits, their activity is not representative of the activity observed in vivo (compare Figs. $7 A, B, 1 A, B$,). Therefore, to test the relevance of our hypothesis for intact functional circuits, we performed additional analysis on 15 min spike-sorted spontaneous V1 activity recorded in vivo under light anesthesia from 140 isolated units in Macaca cyclopsis (Chu et al., 2014). We performed model fitting and FIM sparsity analysis following the same protocol as used for the cultured neurons. As illustrated in Figure $7 C$, model fits were very accurate in predicting the activity statistics, more so in fact than the in vitro maximum entropy models. This result likely reflects the effect of bursting observed in cultures, which leads to excess synchrony. We then computed the FIMs for each group and performed their eigenvector decomposition. The distributions of eigenvalues indicated that the models were sloppy (Fig. 7D), similarly to the models of cultured neuronal activity. However, as illustrated by the comparison of Gini coefficients of model FIMs (Fig. 7E), there was a significant difference in sparsity between the in vivo and in vitro data. As with model fit quality, this effect likely reflects the difference in synchrony between those two types of data, with cortical recordings being more asynchronous and spanning a wider range of firing frequencies. This asynchrony, however, was significantly different from what would be expected of an independently firing population (Fig. 7E). Thus it appears that in cortical networks fewer of the connections are significant than in cultures, and stiff dimensions are even more aligned with the original parameters. Finally, 
we investigated the relationship between firing rates and sensitivity of individual parameters. As illustrated in Figure $7 E$, the trend observed in cultures of high dependency of sensitivity on firing rates is preserved also in vivo. This would suggest that also in vivo the synaptic connections between highly active neurons are the most tightly regulated and most relevant for patterning the network activity.

\section{Sloppiness and sparsity in}

simulated networks

To further understand the interplay of sloppiness and sparsity in different models we also performed our analysis on simulated neural activity. We used randomly connected sparse networks of excitatory and inhibitory leaky integrate and fire neurons (LIF; see Materials and Methods for details) with increasing excitatory synaptic strengths, where the activity becomes progressively more correlated and synchronized (Fig. 8A,B). This analysis underscores the importance of examining not only how sloppy the spectrum of the decomposed FIM is, but also the sparsity of the matrix.

As shown in Figure 8C, increasing the synchrony in the simulated data results in eigenvalues that decrease rapidly in the first few ranks, and then flatten out. In contrast, less synchrony is associated with less prominent dominance of first eigenvector over others. This apparent anisotropy in dimensions in parameter space should not, however, be confused with reliance on few parameters only. Although the first eigenvector of the strongly correlated network is "stiffer" than for other simulated networks, it is not guaranteed to be sparse and have its sensitivity aligned with original parameter dimensions. In Figure $8 D$ it becomes apparent that the trend is, in fact, the opposite. Comparison of Gini coefficients of model FIMs indicates that the more synchronous the activity, the less sparse the model FIMs are. An illustrative example in Figure $8 E$ clarifies why this is the case. For the most synchronous LIF network, the activity of neurons is more homogeneous and all neurons exhibit very similar behavior. As a result, the stiff dimension is composed of a combination of nearly all individual parameters. In the least synchronous LIF network, neurons are on the other hand more differentiated and influence of some parameters is more prominent than others.

Although the LIF model was not intended here to reproduce the different types of data, only as a very simplified analogy, it provides interesting suggestions for interpreting our results and inspiration for further research. The decreasing sparsity in our LIF models (Fig. 8D) mimics the decreasing sparsity in the data (Fig. 7E, "in vivo," "in vitro," and " $>1 \mathrm{~Hz}$ "). The accompanying increase in correlations both in LIF models (Fig. 8B) and in the data (Fig. $7 B$ ) falls in line with the expectation that increased synchrony between neurons will result in more uniform behavior and less differentiation in sensitivity. Because the LIF networks varied only in the excitatory connection strength, this suggests that the cultured neurons might exhibit stronger, on average, synaptic strengths than encountered in vivo. However, it has to be noted that the behavior of rates is different in the simulated data and in recordings, and more detailed and biologically realistic simulations are needed for firmer conclusions.

\section{Discussion}

Here we demonstrate that cultured hippocampal neurons and in vivo cortical networks are well described as sloppy systems. We present a novel framework for stability analysis in application to neurons, which combines two approaches, maximum entropy modeling (Schneidman et al., 2006; Ohiorhenuan et al., 2010) and FIM analysis (Machta et al., 2013). We further show that, in cultured neurons, the activity and coactivity of single neurons undergo considerable spontaneous fluctuations, and these tend to occur along sloppy dimensions in parameter space on the time scale of hours and tens of hours. In contrast, changes along stiff dimensions become prominent only at longer time intervals of days. Finally, we show that sensitivity to changes is highly correlated with firing rate, both in vitro and in vivo. Based on these results we propose that a relatively sparse subnetwork of stable highly active neurons could be supporting operational and possibly functional stability of neuronal networks (Mizuseki and Buzsáki, 2013; Cossell et al., 2015). At the same time, plasticity can take place along sloppy dimensions without compromising stability, thus equipping the network with considerable flexibility to remodel. This is, to our knowledge, the first evidence of sloppiness from models directly fit to neuronal data. Together with 
our findings on sparsity, this might be related to the anisotropy in neuronal representation reported by Hung et al. (2014). Moreover, these results mirror the degeneracy discovered in single neuron expression profiling indicating sloppiness also at the single neuron level (Schulz et al., 2006), which can result in significant flexibility in maintaining neural phenotypes (Prinz et al., 2004).

The particular choice of the parametric description of group behavior was motivated by the fact that pairwise maximum entropy models offer a meaningful, readily interpretable description of neuronal data. The fitted distributions capture the probabilities of binary spike patterns, and thus measure the capacity of the network to code information in those patterns. Furthermore, parameters correspond to local functional properties of neurons and their interactions. However, although the models exactly reproduce the pairwise correlations and individual firing, they fail to account for the full higher-order correlation and temporal structure in the activity (Ohiorhenuan et al., 2010; Yu et al., 2011), and tend to overestimate multineuron firing probabilities (Tkačik et al., 2014). Although we obtained good fits that could account for a large fraction of the correlation structure (Fig. $3 B$ ), the small networks used here are not informative about the relative importance of higher-order interactions in the full circuit (Roudi et al., 2009). Yet inferences about higher order structure in an entire population are generally limited, both computationally, because probability distributions grow exponentially with the number of neurons, and by the availability of data, particularly in the case of sparse activity as observed here. Although full analysis for bigger group sizes is not feasible, it is still possible to compute FIMs directly from the data correlations (see Materials and Methods). A comparison of different group sizes up to 40 neurons showed no systematic differences to the results reported here (not illustrated); we note however, that this data-driven approach implicitly ignores the problem of higher-order correlations, and therefore it can only be viewed as an extension of our results. It is an interesting question whether the same approach could be exploited to narrow down the parameter space and aid the interpretation of larger spatiotemporal models (Marre et al., 2009; Cofré and Cessac, 2014).

Our findings are in part based on analysis of spontaneously active and randomly wired cultured networks, which enabled us to reliably and repeatedly perform simultaneous recordings from hundreds to thousands of neurons with high spatial resolution. This served to avoid the sampling bias expected from conventional recording methods, where typically neurons with low activity are not reliably detected and the very few highly active neurons are undersampled (Mizuseki and Buzsáki, 2013; Wohrer et al., 2013). Moreover, the use of cultured preparations allowed us to reliably track single neurons over days, which was crucial for the purpose of assessing individual fluctuations. While different from the highly structured networks of the cerebral cortex or hippocampus, cultures on the other hand provide insight into the innate properties of self-organized circuits. To test the generality of our findings, we also analyzed an in vivo recording from the visual cortex and confirmed the presence of sloppiness and sparsity in functional networks. Thus, our results suggest that sloppiness and sparsity could be basic inherent properties of neural networks, which are also used as needed in the intact brain. Whether fluctuations over time follow the same principles we discovered in cultured neurons has to be investigated in stable long-term in vivo recordings.

In parallel to observations from cultures (Maletic-Savatic et al., 1999; Okabe et al., 1999; Minerbi et al., 2009), the adult cortex appears to slowly, but continuously remodel its connectivity with variable rates (Trachtenberg et al., 2002; Holtmaat et al., 2005). These changes resemble our observation that the rate of change of the coactivity of single neurons varied substantially. Furthermore, in vivo imaging has shown that spine size fluctuations could be modeled as an Ornstein-Uhlenbeck stochastic process (Loewenstein et al., 2011), which could also account for changes in our data. It is, however, unclear to what extent functional and structural connectivity are related, so more pertinent are the examples from a functional perspective. First, chronic recordings in motor cortex also revealed changing firing rates of individual neurons over the course of days, which however had little influence on decoding performance (Chestek et al., 2007). Second, chronic imaging showed that selectivity of place cells is not generally, but only in subsets of neurons, stable over time, but decoding from the population was again stable over time (Ziv et al., 2013). Both of these studies are consistent with our finding that single neurons can change their activity substantially along insensitive dimensions, without significantly influencing the population activity. Finally, chronic imaging has shown that a small fraction of highly active and selective neurons in barrel cortex were particularly stable over time (Margolis et al., 2012), resembling our finding that highly active neurons and neuron pairs had the highest parameter sensitivity and exhibited only slow remodeling. Together, these results are consistent with the hypothesis that few highly active neurons are critical for stable functional representations.

Thus, although neural coding is typically sparse and distributed and relies on neurons with low activity and high selectivity, the collective functional representations might be held stable by a backbone of highly active, functionally interacting, and perhaps less-selective neurons (Mizuseki and Buzsáki, 2013). Our results predict that these stiff neurons have higher spontaneous rates, and their synapses are less susceptible to plasticity. Such neurons may correspond to a relatively small number of "choristers," which preferentially respond to stimulus categories in primate IT (Hung et al., 2014), and may inherit correlations through direct synaptic contacts (Cossell et al., 2015). Because these neurons play an important role in shaping the overall pattern repertoire of the circuit, changes in their connections are predicted to have a disproportionally large influence on the network response. The remaining sloppy neurons (the "soloists"), on the other hand, are likely much more susceptible to plasticity, and may change their activity more rapidly, for instance to explore a large region of parameter space without a strong effect on collective dynamics and stability. What mechanisms underlie these differences is not clear; a possibility is the weaker LTP reported in neurons with stronger background conductances (Delgado et al., 2010), which may be present in the more highly active stiff neurons. We speculate that this flexibility increases the ability of networks to learn or adapt to novel inputs, because it opens paths between regions in parameter space that would be unavailable in networks with a uniformly high-parameter sensitivity. This form of enhanced evolvability through exploitation of sloppy parameter space domains may be a general property of biological systems (Daniels et al., 2008).

\section{References}

Berdondini L, Imfeld K, Maccione A, Tedesco M, Neukom S, Koudelka-Hep M, Martinoia S (2009) Active pixel sensor array for high spatiotemporal resolution electrophysiological recordings from single cell to large scale neuronal networks. Lab Chip 9:2644-2651. CrossRef Medline

Brown KS, Sethna JP (2003) Statistical mechanical approaches to models with many poorly known parameters. Phys Rev E Stat Nonlin Soft Matter Phys 68:021904. CrossRef Medline 
Carmena JM, Lebedev MA, Henriquez CS, Nicolelis MA (2005) Stable ensemble performance with single-neuron variability during reaching movements in primates. J Neurosci 25:10712-10716. CrossRef Medline

Chestek CA, Batista AP, Santhanam G, Yu BM, Afshar A, Cunningham JP, Gilja V, Ryu SI, Churchland MM, Shenoy KV (2007) Single-neuron stability during repeated reaching in macaque premotor cortex. J Neurosci 27:10742-10750. CrossRef Medline

Chu C, Chien PF, Hung CP (2014) Tuning dissimilarity explains short distance decline of spontaneous spike correlation in macaque $\mathrm{v} 1$. Vision Res 96:113-132. CrossRef Medline

Cofré R, Cessac B (2014) Exact computation of the maximum-entropy potential of spiking neural-network models. Phys Rev E Stat Nonlin Soft Matter Phys 89:052117. CrossRef Medline

Cossell L, Iacaruso MF, Muir DR, Houlton R, Sader EN, Ko H, Hofer SB, Mrsic-Flogel TD (2015) Functional organization of excitatory synaptic strength in primary visual cortex. Nature 518:399-403. CrossRef Medline

Crooks GE (2007) Measuring thermodynamic length. Phys Rev Lett 99: 100602-100606. CrossRef Medline

Daniels BC, Chen YJ, Sethna JP, Gutenkunst RN, Myers CR (2008) Sloppiness, robustness, and evolvability in systems biology. Curr Opin Biotech 19:389-395. CrossRef Medline

Daoudal G, Debanne D (2003) Long-term plasticity of intrinsic excitability: learning rules and mechanisms. Learn Mem 10:456-465. CrossRef Medline

Delgado J, Gómez-González J, Desai NS (2010) Pyramidal neuron conductance state gates spike-timing-dependent plasticity. J Neurosci 30:1571315725. CrossRef Medline

Desai NS, Rutherford LC, Turrigiano GG (1999) Plasticity in the intrinsic excitability of cortical pyramidal neurons. Nat Neurosci 2:515-520. CrossRef Medline

Erickson J, Tooker A, Tai YC, Pine J (2008) Caged neuron mea: a system for long-term investigation of cultured neural network connectivity. J Neurosci Methods 175:1-16. CrossRef Medline

Fitzsimonds RM, Song HJ, Poo MM (1997) Propagation of activitydependent synaptic depression in simple neural networks. Nature 388: 439-448. CrossRef Medline

Fusi S, Abbott LF (2007) Limits on the memory storage capacity of bounded synapses. Nat Neurosci 10:485-493. CrossRef Medline

Goodman DF, Brette R (2009) The brain simulator. Front Neurosci 3:192197. CrossRef Medline

Holtmaat AJ, Trachtenberg JT, Wilbrecht L, Shepherd GM, Zhang X, Knott GW, Svoboda K (2005) Transient and persistent dendritic spines in the neocortex in vivo. Neuron 45:279-291. CrossRef Medline

Huber D, Gutnisky DA, Peron S, O’Connor DH, Wiegert JS, Tian L, Oertner TG, Looger LL, Svoboda K (2012) Multiple dynamic representations in the motor cortex during sensorimotor learning. Nature 484:473-478. CrossRef Medline

Hung CP, Cui D, Chen Y-p, Lin C-p, Levine MR (2014) Correlated activity supports efficient cortical processing. Front Comput Neurosci 8:1-16.

Hurley N, Rickard S (2009) Comparing measures of sparsity. IEEE T Inform Theory 55:4723-4741. CrossRef

Lesuisse C, Martin LJ (2002) Long-term culture of mouse cortical neurons as a model for neuronal development, aging, and death. J Neurobiol 51:9-23. CrossRef Medline

Loewenstein Y, Kuras A, Rumpel S (2011) Multiplicative dynamics underlie the emergence of the log-normal distribution of spine sizes in the neocortex in vivo. J Neurosci 31:9481-9488. CrossRef Medline

Machta BB, Chachra R, Transtrum MK, Sethna JP (2013) Parameter space compression underlies emergent theories and predictive models. Science 342:604-607. CrossRef Medline

Maletic-Savatic M, Malinow R, Svoboda K (1999) Rapid dendritic morphogenesis in cal hippocampal dendrites induced by synaptic activity. Science 283:1923-1927. CrossRef Medline

Margolis DJ, Lütcke H, Schulz K, Haiss F, Weber B, Kügler S, Hasan MT, Helmchen F (2012) Reorganization of cortical population activity imaged throughout long-term sensory deprivation. Nat Neurosci 15:15391546. CrossRef Medline

Marre O, El Boustani S, Frégnac Y, Destexhe A (2009) Prediction of spatio- temporal patterns of neural activity from pairwise correlations. Phys Rev Lett 102:138101. CrossRef Medline

Minerbi A, Kahana R, Goldfeld L, Kaufman M, Marom S, Ziv NE (2009) Long-term relationships between synaptic tenacity, synaptic remodelling, and network activity. PLoS Biol 7:e1000136(1-20). Medline

Mizuseki K, Buzsáki G (2013) Preconfigured, skewed distribution of firing rates in the hippocampus and entorhinal cortex. Cell Rep 4:1010-1021. CrossRef Medline

Ohiorhenuan IE, Mechler F, Purpura KP, Schmid AM, Hu Q, Victor JD (2010) Sparse coding and high-order correlations in fine-scale cortical networks. Nature 466:617-621. CrossRef Medline

Okabe S, Kim HD, Miwa A, Kuriu T, Okado H (1999) Continual remodeling of postsynaptic density and its regulation by synaptic activity. Nat Neurosci 2:804-811. CrossRef Medline

Prinz AA, Bucher D, Marder E (2004) Similar network activity from disparate ciruit parameters. Nat Neurosci 7:1345-1352. CrossRef Medline

Quiroga RQ, Nadasdy Z, Ben-Shaul Y (2004) Unsupervised spike detection and sorting with wavelets and super-paramagnetic clustering. Neural Comput 16:1661-1687. CrossRef Medline

Roudi Y, Nirenberg S, Latham PE (2009) Pairwise maximum entropy models for studying large biological systems: when they can work and when they can't. PLoS Comput Biol 5:e1000380. CrossRef Medline

Schneidman E, Berry MJ 2nd, Segev R, Bialek W (2006) Weak pairwise correlations imply strongly correlated network states in a neural population. Nature 440:1007-1012. CrossRef Medline

Schulz DJ, Goaillard JM, Marder E (2006) Variable channel expression in identified single and electrically coupled neurons in different animals. Nat Neurosci 9:356-362. CrossRef Medline

Segev R, Baruchi I, Hulata E, Ben-Jacob E (2004) Hidden neuronal correlations in cultured networks. Phys Rev Lett 92:118102. CrossRef Medline

Shlens J, Field GD, Gauthier JL, Grivich MI, Petrusca D, Sher A, Litke AM, Chichilnisky EJ (2006) The structure of multi-neuron firing patterns in primate retina. J Neurosci 26:8254-8266. CrossRef Medline

Slomowitz E, Styr B, Vertkin I, Milshtein-Parush H, Nelken I, Slutsky M, Slutsky I (2015) Interplay between population firing stability and single neuron dynamics in hippocampal networks. Elife 4:e04378. CrossRef Medline

Tang A, Jackson D, Hobbs J, Chen W, Smith JL, Patel H, Prieto A, Petrusca D, Grivich MI, Sher A, Hottowy P, Dabrowski W, Litke AM, Beggs JM (2008) A maximum entropy model applied to spatial and temporal correlations from cortical networks in vitro. J Neurosci 28:505-518. CrossRef Medline

Tkačik G, Marre O, Amodei D, Schneidman E, Bialek W, Berry MJ 2nd (2014) Searching for collective behavior in a large network of sensory neurons. PLoS Comput Biol 10:e1003408. CrossRef Medline

Trachtenberg JT, Chen BE, Knott GW, Feng G, Sanes JR, Welker E, Svoboda $\mathrm{K}$ (2002) Long-term in vivo imaging of experience-dependent synaptic plasticity in adult cortex. Nature 420:788-794. CrossRef Medline

Turrigiano G (2011) Too many cooks? Intrinsic and synaptic homeostatic mechanisms in cortical circuit refinement. Annu Rev Neurosci 34:89103. CrossRef Medline

van Pelt J, Vajda I, Wolters PS, Corner MA, Ramakers GJ (2005) Dynamics and plasticity in developing neuronal networks in vitro. Prog Brain Res 147:173-188. Medline

van Rossum MC, Bi GQ, Turrigiano GG (2000) Stable hebbian learning from spike timing-dependent plasticity. J Neurosci 20:8812-8821. Medline

Wagenaar DA, Pine J, Potter SM (2006) An extremely rich repertoire of bursting patterns during the development of cortical cultures. BMC Neurosci 7:11. CrossRef Medline

Wohrer A, Humphries MD, Machens CK (2013) Population-wide distributions of neural activity during perceptual decision-making. Prog Neurobiol 103:156-193. CrossRef Medline

Yu S, Yang H, Nakahara H, Santos GS, Nikolić D, Plenz D (2011) Higherorder interactions characterized in cortical activity. J Neurosci 31:17514 17526. CrossRef Medline

Ziv Y, Burns LD, Cocker ED, Hamel EO, Ghosh KK, Kitch LJ, El Gamal A, Schnitzer MJ (2013) Long-term dynamics of cal hippocampal place codes. Nat Neurosci 16:264-266. CrossRef Medline 